\title{
Microquasar Jets: A Comparison with Extragalactic Jets
}

\author{
Ralph Spencer, Chris De La Force \\ Jodrell Bank Observatory, University of Manchester, UK \\ Alastair Stirling \\ University of Central Lancashire, UK
}

\begin{abstract}
We compare the properties of jets found in extragalactic sources with those recently discovered in the much lower luminosity microquasars in our galaxy.
\end{abstract}

\section{Introduction}

Superluminal jets have recently been discovered from X-ray emitting binaries in our own galaxy (Mirabel \& Rodriguez 1999, Fender 2000a). These 'microquasars' seem to be much less luminous version of the more powerful radio galaxies and quasars, however the jets found in both kinds of object are thought to originate via accretion processes onto a compact object: a massive black hole in extragalactic sources and a stellar mass black hole or neutron star in X-ray binaries.

Collimated jets are found in many types of extragalactic radio source (see e.g. Zensus 1997), where there is a need to re-supply energy lost by to radiative losses in lobes and hot spots. Jets dominate radio emission in the lower luminosity FR I sources (L(radio) $<10^{35} \mathrm{~W}$ ), e.g. M87 and $3 \mathrm{C} 120$, whereas lobes and hotspots dominate in higher luminosity FR II objects (e.g. Cygnus A and 3 C295). Motion of knots and features have been detected in jets at velocities of 0.3 to $0.98 \mathrm{c}$, and Doppler boosting gives rise to strong selection effects. Jets range in scale from pc to 100's kpc, with opening angles down to a few degrees. The jet powers range from $10^{38}$ to $10^{40} \mathrm{~W}$ (Lobanov et al. 1998) and in extended sources supply energy to the lobes which accumulates to levels of $10^{53} \mathrm{~J}$.

\section{Microquasars}

Jets in microquasars are dominated by constant velocity (up to $0.95 \mathrm{c}$ ) moving knots or blobs which can be traced back to the original outburst, suggesting ballistic motion (Fender et al. 1999). Angles to line of sight of 30-80 degrees have been derived, so Doppler boosting does not dominate selection effects, as expected since the objects were originally detected on the basis of their presumably unbeamed X-ray emission. Recently continuous jets have been discovered in persistent sources, which may have different properties to the sporadic jet transient sources (Stirling et al. 1998 and 2000, Fender 2000b). Jet sizes range from 10 to 500000 au and kinetic powers range from $10^{27}$ to $10^{30} \mathrm{~W}$, much greater than the jet radio luminosity $\left(\sim 10^{24}\right.$ to $\left.10^{26} \mathrm{~W}\right)$ (Spencer 1996). Given 
that these are only minimum energy estimates and that protons have been ignored then the jet powers are perhaps not much smaller than the total accretion powers.

\section{Comparison between XRB jets and AGN Jets}

The similarities between microquasar and extragalactic source jets (EGJ) can be summarised as follows: 1) EGJ and XRB jets both have a wide range of linear sizes (over factors of $\sim 10^{5}$ ), 2) Well collimated jets are seen in both types of object, 3) They are efficient transporters of energy (their kinetic luminosities are $\sim 10^{3}$ larger than their radiative losses), 4) Knots and continuous jets are seen in both kinds of object.

The differences are: 1) XRB jets have powers of $10^{30}$ to $10^{33} \mathrm{~W}$ (including a contribution from protons), $\sim 10^{6}$ times smaller than for AGN jets; 'Microquasars' is a good name!, 2) Major ejections in the sporadic XRB sources show ballistic motion whereas EGJ show variations in velocity and direction of motion along the jet due to instabilities and external forces, 3) It is unclear if XRB jets have a non-radiating underlying jet always present or not, 4) Radiative lifetimes are long in XRB jets so radiation losses are unimportant for the radio emitting electrons, though self-Compton radiation may be important for the compact $\mathrm{XRB}$ jets, 5) Unlike the EGJ there is no clear evidence for the knots in XRB jets being due to shock compression, 6) There is no evidence for significant dissipation regions for the energy in XRB jets, unlike the lobes and hot-spots of AGN.

\section{Unresolved problems}

1) What is the role of protons in XRB jets? Perhaps polarization studies can help as for AGN.

2) What happens to the kinetic energy in XRB jets, since interaction regions are not seen; where is Scheuer's waste energy basket?

3) Are the jets in XRBs always ballistic, if so why should this be different to that in AGN jets?

\section{References}

Fender, R. P. 2000a, in Astrophysics and Cosmology, Springer (astro-ph/9907050) Fender, R. P. 2000b, MNRAS, in press

Fender, R.P., et al. 1999, MNRAS, 304, 865

Lobanov, A. et al. 1998, A\&A, 340, 79

Mirabel, I. F., Rodriguez, L. F. 1999, ARA\&A, 37, 409

Spencer, R. E. 1996, in Cataclysmic Variables and Related Objects. IAU 128, eds A. Evans and J. Wood, 371, Kluwer

Stirling, A., et al. 1998 New AR, 42, 657

Stirling, A., et al. 2000 in prep.

Zensus, J. A. 1997, ARA\&A, 35, 607 